CERN-ECP/95-10

18 May 1995

\title{
A TRANSIMPEDANCE AMPLIFIER USING A NOVEL CURRENT MODE FEEDBACK LOOP
}

\author{
F. Anghinolfi'1), W. Dabrowski ${ }^{3)}$, E. Delagnes ${ }^{2)}$, P. Jarron ${ }^{1)}$, L. Scharfetter ${ }^{1)}$
}

\begin{abstract}
We present a transimpedance amplifier stage based on a novel current mode feedback topology. This circuit employs NMOS and PMOS transistors exclusively and requires neither capacitor for stabilizing the transimpedance loop nor resistor for the transresistance feedback and transistor loading. This amplifier circuit is fully compatible with submicron digital CMOS processes. The active feedback network consists of two grounded-gate MOS devices which split the output current in both the feedback and output branches. The transresistance and the phase margin are adjustable through external DC signals. The measured rise time of the impulse response of the amplifier implemented in an industrial $0.7 \mu \mathrm{m}$ CMOS process is $18 \mathrm{~ns}$ for a transresistance of $180 \mathrm{k} \Omega$ and $30 \mathrm{~ns}$ for a transresistance of $560 \mathrm{k} \Omega$. The measured Equivalent Noise Charge (ENC) is $800 \mathrm{rms} \mathrm{e}^{-}$for an input capacitance of $20 \mathrm{pF}$ with the transresistance adjusted to $560 \mathrm{k} \Omega$.
\end{abstract}

Presented at the 7th European Symposium on semiconductor detectors, Schloss Elmau, Germany, 7-10 May 1995. To be published in NIM A

1) CERN, Geneva, Switzerland.

2) CEA, DSM/DAPNIA, CE-Saclay, F-91191 GIF-sur-Yvette Cedex, France.

3) Faculty of Physics and Nuclear Techniques, Cracow, Poland. 



\section{INTRODUCTION}

Transimpedance amplifier designs with low-noise and high-speed characteristics are key components in many front-ends, such as optical data-link receivers, smart-pixel-sensor amplifiers and preamplifiers for radiation detectors. The transimpedance configuration circuit is also an attractive approach for fast amplifiers for silicon-drift and -strip detectors at the LHC experiments. Several of the current sensitive preamplifiers for silicon-strip detector applications have been designed in hybrid circuits with bipolar [1] and MOS [2] technologies. More recently, transimpedance preamplifiers have been designed in bipolar, integrated-circuit technologies [3-7].

The main features desired for fast front-end amplifiers are low noise, high gain and excellent frequency stability. Moreover, the developement of front-end systems in radiationhard CMOS processes [8-10] for the future LHC detectors and advances in deep submicron CMOS technology require design circuit techniques increasingly compatible with digital CMOS processes. The active current feedback circuit principle presented in this paper provides a means of improving the speed performance of a transimpedance amplifier without sacrificing stability or noise, and is fully compatible with digital processes. This novel circuit technique is very compact and enables control of the transresistance gain over a large range (1-10), via an external current, again without affecting amplifier stability. The amplifier architecture can accept a DC input connection with a leaky sensor, such as a silicon-strip detector, without its performance being impared. This circuit does not require any additional passive components such as capacitors or resistors and is therefore compatible with deep submicron CMOS processes. Alternatively, the same circuit can be used to implement a highvalue feedback resistor ( $>10 \mathrm{M} \Omega$ ) for a charge amplifier, by operating the active feedback at very low current.

The active feedback loop circuit principle is presented in Section 2, and is compared with the traditional transresistance feedback structure. In Section 3 the implementation of the active feedback in a transimpedance amplifier in CMOS technology is presented and crucial design issues are discussed. The first experimental results of the active feedback transimpedance amplifier are presented in Section 4, in particular, the noise characteristics. Section 5 concludes the paper with discussion of the future development of this circuit.

\section{THE ACTIVE FEEDBACK PRINCIPLE}

Figure 1 shows a basic circuit diagram for a traditional CMOS or bipolar transimpedance amplifier. Low-noise performance is ultimately determined by the parallel input noise current, inversely proportional to $R_{f}$. Hence, from the point of view of noise, a high value of $R_{f}$ is desired - typically above $100 \mathrm{k} \Omega$ - to keep the parallel Equivalent Noise Charge (ENC) contribution below $500 \mathrm{rms}$ electrons for a system peaking time of 25 $\mathrm{ns}$ to $50 \mathrm{~ns}$. One important consideration is the maintenance of stability under all operating conditions. Major parasitic capacitances affecting the frequency response of the amplifier are shown in Fig. 1. Assuming that the Miller effect of $\mathrm{Cm}$ is negligible, the dominant and second poles are given by:

$$
\omega_{\mathrm{d}} \approx \frac{\mathrm{A}_{0}}{\mathrm{C}_{\mathrm{IN}} \mathrm{R}_{\mathrm{f}}} \quad \text { and } \quad \omega_{\mathrm{N}} \approx \frac{1}{\mathrm{C}_{\mathrm{L}} \mathrm{R}_{\mathrm{L}}}
$$




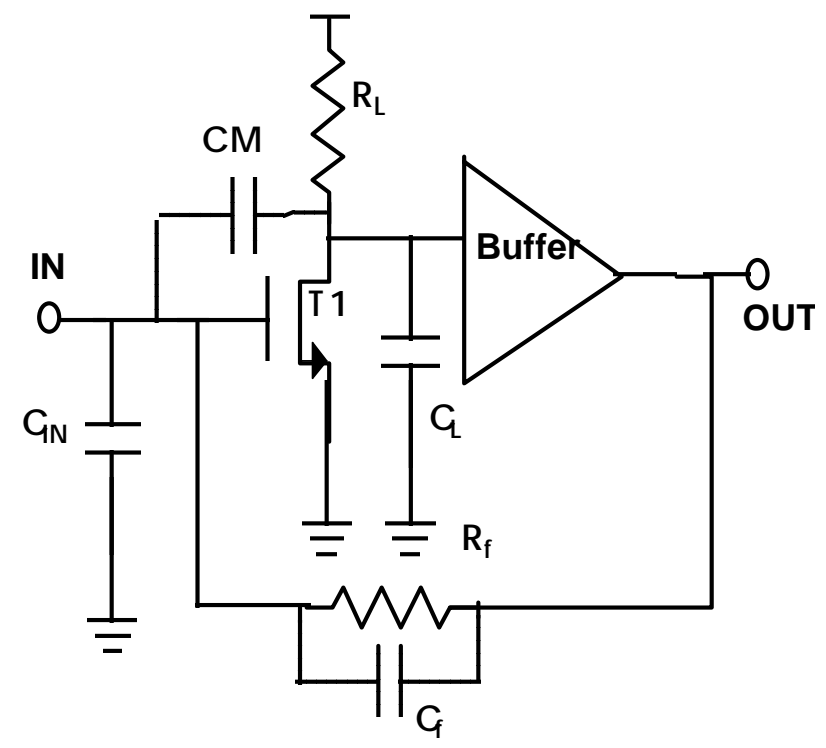

Fig. 1 Traditional transimpedance amplifier using a feedback resistor. The input device T1, here a MOS transistor, is also in several designs a bipolar transistor. An additional frequency compensation by $C_{f}$ is commonly used to improve stability, at the expense of a gain-bandwidth reduction.

where $A_{0}$ is the open loop voltage gain, and $C_{I N}$ represents the detector capacitance, amplifier capacitance and the interconnected parasitic capacitance.

The stability of the amplifier modelled with a two-pole transfer function imposes a minimum phase margin of $58^{\circ}$ and an ideal one of more than $76^{\circ}$, requiring $\omega_{d}$ to be at least 2.7 times larger than $\omega_{N}$. In practice, such an amplifier design requires an additional feedback compensation capacitor to add a right-half-plane zero, in order to keep the stability unaffected by the variations of $R_{f}, R_{L}$ and $C_{I N}$. The drawback of this compensation is a reduction of the amplifier gain-bandwidth product.

This problem is addressed by using an active feedback network based on two MOS devices, $\mathrm{MP}_{f}$ and $\mathrm{MP}_{0}$, as shown in Fig. 2. The transistor $\mathrm{MP}_{f}$ is placed in the feedback path of the transconductance amplifier, A, where the conventional feedback resistor and transistor $\mathrm{MP}_{0}$ loads the output node OUTV. Transistor $\mathrm{MP}_{f}$ is in saturation and is biased close to weak inversion by the current source $\mathrm{MI}_{f}$. Therefore, $\mathrm{MP}_{f}$ acts as a cascode stage across the feedback path so that it replaces the feedback resistor $R_{f}$ and the load resistor $R_{L}$, and does not require the buffer depicted in Fig.1. It also maintains adequate biasing conditions for the amplifier A. An additional advantage is that it provides a supplementary current output, OUTI.

The transconductance $g m s_{f}$ determines the effective feedback resistor $R_{f}$, the effective load resistor $R_{L}$ and the mid-band gain $A_{0}$, which can be expressed for weak inversion operation as:

$$
\mathrm{R}_{\mathrm{f}}=\frac{1}{\mathrm{gms}_{\mathrm{f}}}, \quad \mathrm{R}_{\mathrm{L}}=\frac{1}{\mathrm{gms}_{\mathrm{f}}}, \quad \mathrm{A}_{0}=\frac{\mathrm{gm}_{1}}{\mathrm{gms}_{\mathrm{f}}} \quad \text { with } \quad \mathrm{gms}_{\mathrm{f}}=\frac{\mathrm{q}}{\mathrm{kT}} \mathrm{I}_{\mathrm{f}}
$$

where $k$ is the Boltzman constant and $T$ the absolute temperature.

Thus, the dominant and the non-dominant poles become: 


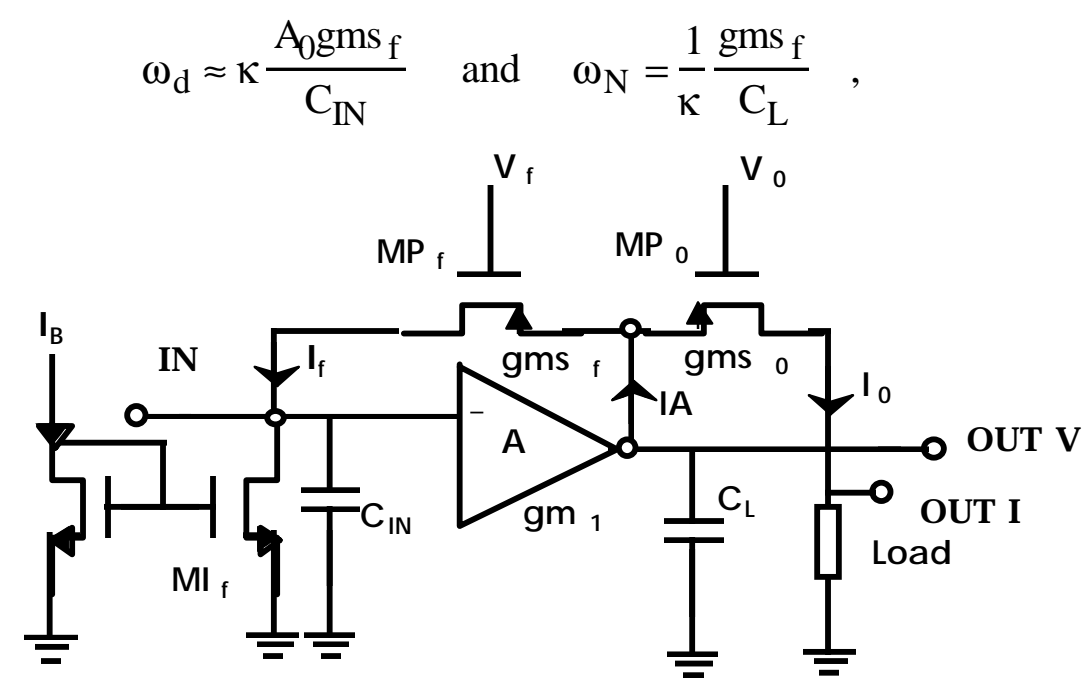

Fig. 2 Circuit principle of the active feedback loop. $\mathrm{MP}_{f}$ acts as a feedback resistor adjustable via $I_{B}$. $\mathrm{MP}_{0}$ provides the phase margin adjustment. The amplifier has two outputs: OUTV, with a high transresistance gain and small dynamic range; and OUTI, with a large dynamic range and current gain $\mathrm{gms}_{\mathrm{O}} / \mathrm{gms}_{\mathrm{f}}$.

where $\kappa=\left(\mathrm{gms}_{\mathrm{f}}\right) /\left(\mathrm{gms}_{\mathrm{f}}+\mathrm{gms}_{0}\right)$. One can note that both poles can be controlled by $\kappa$. If one assumes that the transistor pair formed by $\mathrm{MP}_{f}$ and $\mathrm{MP}_{0}$ works in weak inversion, the drain currents and $\kappa$ can be expressed as [8]:

$$
\begin{gathered}
I_{f}=I_{s a t} e^{-\left(V_{f} q / n k T\right)}\left[e^{\left(V_{s} q / n k T\right)}-e^{\left(V_{d} q / n k T\right)}\right], \\
I_{0}=I_{s a t} e^{-\left(V_{0} q / n k T\right)}\left[e^{\left(V_{s} q / n k T\right)}-e^{\left(V_{d} q / n k T\right)}\right],
\end{gathered}
$$

with

$$
\kappa=\frac{1}{1+e^{-\left[\left(V_{0}-V_{f}\right) q / n k T\right]}}
$$

where $I_{s a t}$ is the saturation drain current, $V_{s}$ the voltage of the output node and $V_{d}$ the drain voltage of $\mathrm{MP}_{f}$ and $\mathrm{MP}_{0}$.

Therefore, the phase margin controlled by $\kappa$ can be adjusted by the differential voltage $V_{0}-V_{f}$.

Assuming that the zeros introduced by Miller capacitances and the internal pole of the stage $\mathrm{A}$ are negligible, the gain of the transconductance amplifier can be modelled as a second-order system which can be expressed by a two-pole transfer function:

$$
\mathrm{G}(\mathrm{s})=\frac{\mathrm{V}_{\mathrm{out}}(\mathrm{s})}{\mathrm{I}_{\mathrm{in}}} \approx-\frac{1}{\mathrm{gms}_{\mathrm{f}}} \frac{1}{\mathrm{~s}^{2} \frac{\mathrm{C}_{\mathrm{IN}} \mathrm{C}_{\mathrm{L}}}{\mathrm{gm}_{1} \mathrm{gm}_{\mathrm{f}}}+\mathrm{s} \frac{1}{\kappa} \frac{\mathrm{C}_{\mathrm{IN}}}{\mathrm{gm}}+1} .
$$

For a phase margin between $58^{\circ}$ and $76^{\circ}$, the roots of the denominator occur as complex-conjugate pairs. In this condition, the inverse Laplace transform of Eq. (5) for a step response has the following expression:

$$
G(t)=-\frac{1}{g m s_{f}}\left[1-\frac{e^{-\left(\omega_{c} t / 2 \xi\right)}}{\sqrt{1-\left(1 / 4 \xi^{2}\right)}} \sin \left(\omega_{c} t \sqrt{1-\frac{1}{4 \xi^{2}}}+\arcsin \sqrt{1-\frac{1}{4 \xi^{2}}}\right)\right],
$$


where $\xi=\kappa \quad \sqrt{\left(\mathrm{gm}_{1} \mathrm{C}_{\mathrm{L}} / \mathrm{gms}_{\mathrm{f}} \mathrm{C}_{\mathrm{IN}}\right)}$ and $\omega_{\mathrm{c}}=\sqrt{ }\left(\mathrm{gm}_{1} \mathrm{gms}_{\mathrm{f}} / \mathrm{C}_{\mathrm{L}} \mathrm{C}_{\mathrm{IN}}\right)$. The response of the amplifier to an input current impulse obtained by differentiation of Eq. (6) is plotted in Fig. 3 for typical parameters of a $0.7 \mu \mathrm{m}$ CMOS technology and with an equivalent feedback resistance of $300 \mathrm{k} \Omega$. A peaking time of $15 \mathrm{~ns}$ is calculated with a phase margin of $70^{\circ}$ for $C_{I N}=20 \mathrm{pF}$ and $g m_{1}=5 \mathrm{mS}$.

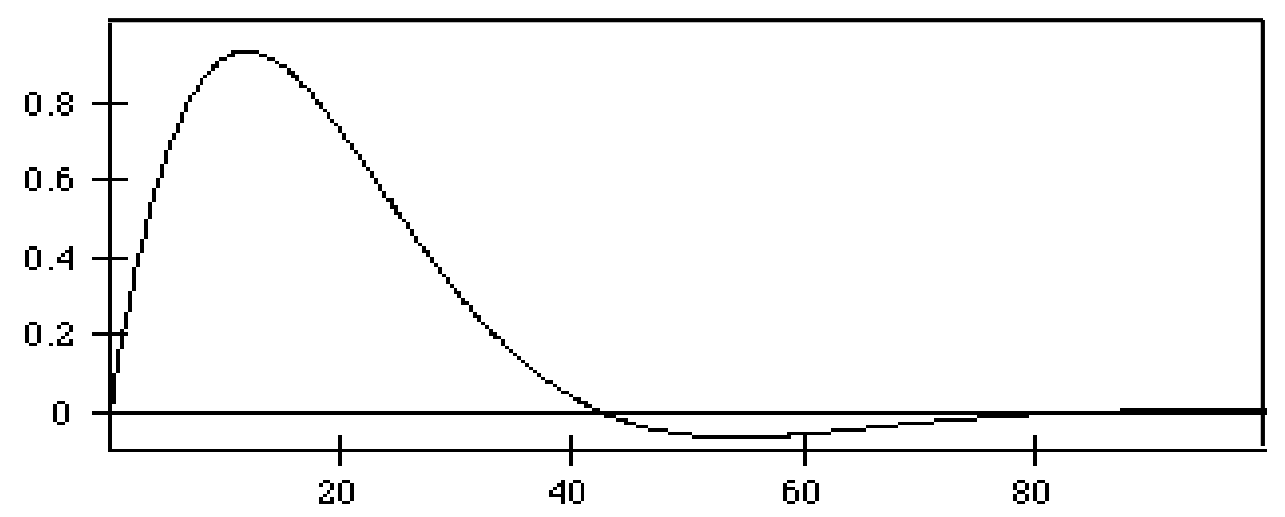

Fig. 3 Output pulse transient response of the active feedback amplifier shown in Fig. 2. The circuit has been modelled for a $0.7 \mu \mathrm{m}$ CMOS technology as a simplified second-order system. $\kappa$ has been adjusted by $V_{f}-V_{O}$ to obtain a phase margin of $70^{\circ}$. Time scale is in ns and amplitude is normalized to 1 .

\section{LOW-NOISE TRANSIMPEDANCE PREAMPLIFIER WITH ACTIVE FEEDBACK}

The practical implementation of the active current feedback circuit is shown in Fig. 4. It uses the direct cascode configuration built with NMOS or NPN bipolar transistors T1 and T2. In the case of MOS devices, the input transistor T1 is sized to match the sensor capacitance for minimum noise, whereas the aspect ratio of the cascode transistor T2 is chosen to reduce the parasitic capacitance on the output node. In the case of bipolar input, the transistor geometry is sized to keep the base-spreading resistance negligible in comparison with the equivalent noise resistance of the collector shot noise.

Here we study only the MOS version. The active current feedback loop is implemented by the PMOS transistor $\mathrm{MP}_{f}$ and the current source $\mathrm{Mi}_{f}$. With the load transistor $\mathrm{MP}_{0}$, transistor $\mathrm{MP}_{f}$ acts as a cascode stage across the feedback loop and loads the output node with its source resistance $1 / g m s_{f} . \mathrm{MP}_{f}$ and $\mathrm{MP}_{O}$ sizes are close to the minimum size $\left(C_{L}\right.$ minimum), in order to keep $\omega_{d}$ as high as possible. The adjustable current source $\mathrm{MI}_{f}$ biases $\mathrm{MP}_{f}$ close to weak inversion for a drain current in the $50 \mathrm{nA}$ to $1 \mu \mathrm{A}$ range. The mid-band input resistance of the active feedback transimpedance amplifier is determined by the ratio $R_{f}$ $/ A_{0}=1 / g m s_{1}$, which is not dependent on $R_{f}$.

The DC operating biasing of the amplifier, shown in Fig. 4, is set by three external voltage sources $\left(V_{C A S}, V_{f}\right.$ and $\left.V_{0}\right)$ and two external current sources $\left(I_{B}\right.$ and $\left.I_{D}\right)$. Drain voltages of $\mathrm{T} 1$ and $\mathrm{T} 2$ are entirely set by gate voltages $V_{f}$ and $V_{C A S}$. The current balance $I_{f}-I_{O}$ is set by $V_{O}$. This biasing scheme enables single-rail, low-supply voltage operation. 


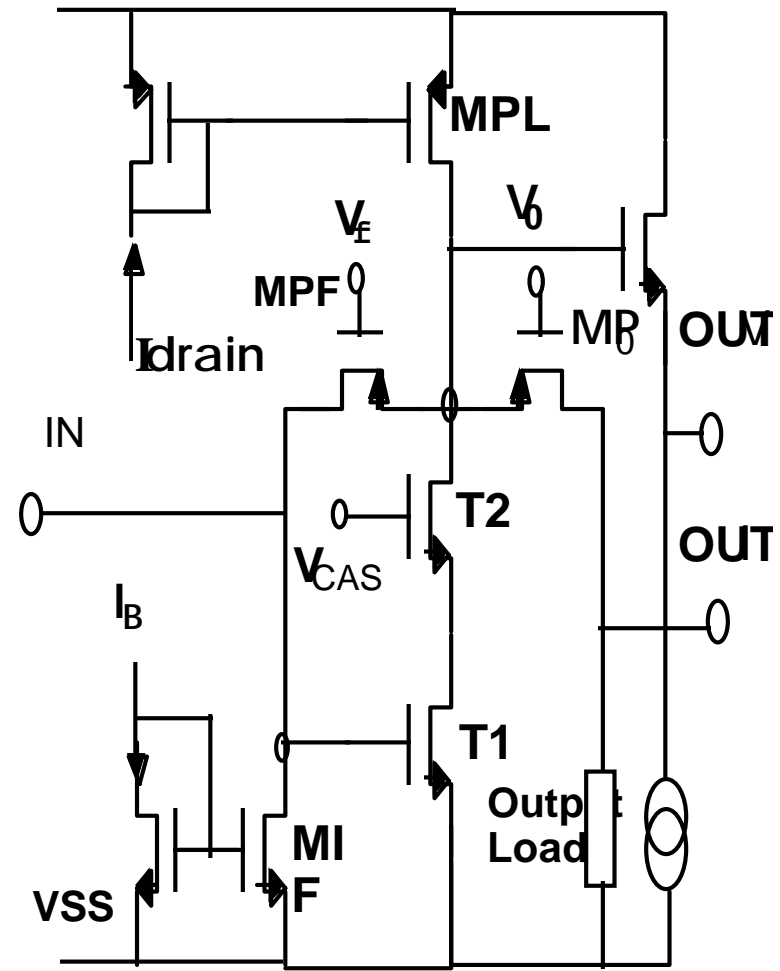

Fig. 4 Circuit diagram of the active feedback transimpedance amplifier in CMOS technology.

The setting and polarity of the input current source $I_{B}$ defines three dynamic modes of amplification for the output OUTV:

1. Linear mode: when the input signal is sufficiently small compared to $I_{B}$, the amplification is practically linear (if $\geq 300 \mathrm{nA}$ and input charge $\leq 12 \mathrm{fC}$ ).

2. Square root compression: when the polarity of the input signal is negative and large compared to $I_{B}, g m s_{f}$ varies like the square root of the input current $\left(\mathrm{MP}_{f}\right.$ in strong inversion). Hence, the amplifier accomplishes a square root compression of the input signal.

3. Non-linear mode: when the polarity of the input signal is positive and $I_{B}$ is sufficiently small $(100 \mathrm{nA})$, the input signal forces the feedback current $I_{f}$ to 0 , switching $\mathrm{MP}_{f}$ off. Then the feedback is opened and the circuit is configured like an open loop transconductance, which enhances the gain considerably (by about a factor of 10). This effect can be used to obtain a non-linear signal processing in such a way that signal and noise level below the switching threshold are dynamically compressed. The technique can be applied to decrease the noise hit rate of readout electronics for a binary or sparse-data-scan-readout system.

The current-output OUTI delivers a linear-output signal with a current gain, $\mathrm{I}_{\mathrm{out}} / \mathrm{I}_{\mathrm{IN}}=$ gms $_{0} / \mathrm{gms}_{\mathrm{f}}$. This output can be used in current mode with a low impedance load or in integrating mode with a capacitive output node, with the current output OUTI working like a charge amplifier output. 
SPICE simulation of the circuit produces the transient impulse response shown in Fig. 5. The simulation indicates a more symmetric pulse shape than the calculated result of Fig. 3. Secondary poles and zeros of the direct cascode amplifier not included in the second-order calculation explain this difference.

Fig. 5 SPICE simulation result: transient output pulse response for $I_{f}=250 \mathrm{nA}$. The drain current of the cascode amplifier is $400 \mu \mathrm{A}$ and $C_{I N}=10 \mathrm{pF}$.

The noise contributions of transistors $\mathrm{MP}_{f}$ and $\mathrm{MP}_{0}$ operating in weak inversion can be expressed from Eq. (2) as the power density of the noise current at the input:

$$
\overline{\mathrm{I}}_{\mathrm{f}}^{2}=\overline{\mathrm{In}}_{\mathrm{I}}^{2}=4 \mathrm{kT} \frac{1}{2} \mathrm{gms}_{\mathrm{f}}=\frac{1}{2} \frac{4 \mathrm{kT}}{\mathrm{R}_{\mathrm{f}}}
$$

Hence, the total parallel input noise current is

$$
\overline{\mathrm{I}}_{\mathrm{f}}^{2}+\overline{\mathrm{I}}_{\mathrm{I}}^{2}=\frac{4 \mathrm{kT}}{\mathrm{R}_{\mathrm{f}}}
$$

which is equal to the noise of a traditional transimpedance amplifier. If the design could be optimized to operate $\mathrm{MP}_{f}$ and $\mathrm{MP}_{0}$ in strong inversion with $g m s_{I}<<g m s_{f}$, the parallel noise would be slightly improved. In this case the total parallel noise current would be: 


$$
\overline{\mathrm{In}}_{\mathrm{f}}^{2}=4 \mathrm{kT} \frac{2}{3} \mathrm{gm}_{\mathrm{f}}=\frac{2}{3} \frac{4 \mathrm{kT}}{\mathrm{R}_{\mathrm{f}}}
$$

Thus, the parallel noise decreases by $30 \%$ when compared to a conventional feedback resistor. Noise analysis is here done assuming that the bulk transconductance associated with the bulk noise resistance of $\mathrm{MP}_{f}$ and $\mathrm{MP}_{0}$ does not contribute to the noise. This assumption is justified because $\mathrm{MP}_{f}$ and $\mathrm{MP}_{0}$ have noise resistance larger than $100 \mathrm{k} \Omega$, which is much higher than the bulk noise contribution of a small geometry transistor.

The series-noise contribution of the active feedback amplifier is essentially identical to the conventional transimpedance or charge amplifier and is mostly determined by the noise characteristics of the input transistor T1. It can be noted that because of its high gain $(10 \mathrm{mV} / \mathrm{fC})$, this configuration exhibits a greater robustness against second-stage noise contribution than a fast charge amplifier.

\section{EXPERIMENTAL RESULTS}

The active feedback transimpedance amplifier circuit of Fig. 4 has been designed and fabricated using industrial $0.7 \mu \mathrm{m}$ CMOS technology 1 ). The T1 input NMOS device has a size of $\mathrm{W} / \mathrm{L}=2000 \mu \mathrm{m} / 1.2 \mu \mathrm{m}$ and is biased at $400 \mu \mathrm{A}$. The $\mathrm{MP}_{f}$ and $\mathrm{MP}_{0}$ transistors have a size of $\mathrm{W} / \mathrm{L}=2 \mu \mathrm{m} / 4 \mu \mathrm{m}$. The preliminary measured output pulse shape response for charge input of $1 \mathrm{MIP}(4 \mathrm{fC})$ is shown in Fig. 6 . The measurement has been performed with a test board presenting a minimum input capacitance of $10 \mathrm{pF}$. In these conditions the active feedback circuit shows an excellent stability. The adjustment of the phase margin with the differential voltage $V_{f}-V_{0}$ has been verified and works as predicted. The results from experimental output pulse responses shown in Fig. 6 fully agree with SPICE simulations.

Fig. 6 Measured output transient pulse response for $I_{f}=120 \mathrm{nA}, 186 \mathrm{nA}, 235 \mathrm{nA}, 580 \mathrm{nA}$. The drain current of the cascode amplifier is $400 \mathrm{~mA}$ and $C_{I N}=10 \mathrm{pF}$. Rise time varies from $18 \mathrm{~ns}$ to $30 \mathrm{~ns}$. 
The transresistance of the active feedback circuit has been measured as shown in Fig. 7. The variation of the transresistance by a factor of 3 from $190-580 \mathrm{k} \Omega$ is obtained for an $I_{B}$ variation of a factor 4.7 , from 580-120 nA. This result is slightly different from the calculation of Eq. (2) which predicts a linear dependence. The reason is that the transistor $\mathrm{MP}_{f}$, for $I_{B}$ above $200 \mathrm{nA}$, begins to operate in moderate inversion.

1) Mietec NV, Oudenaarde, Belgium

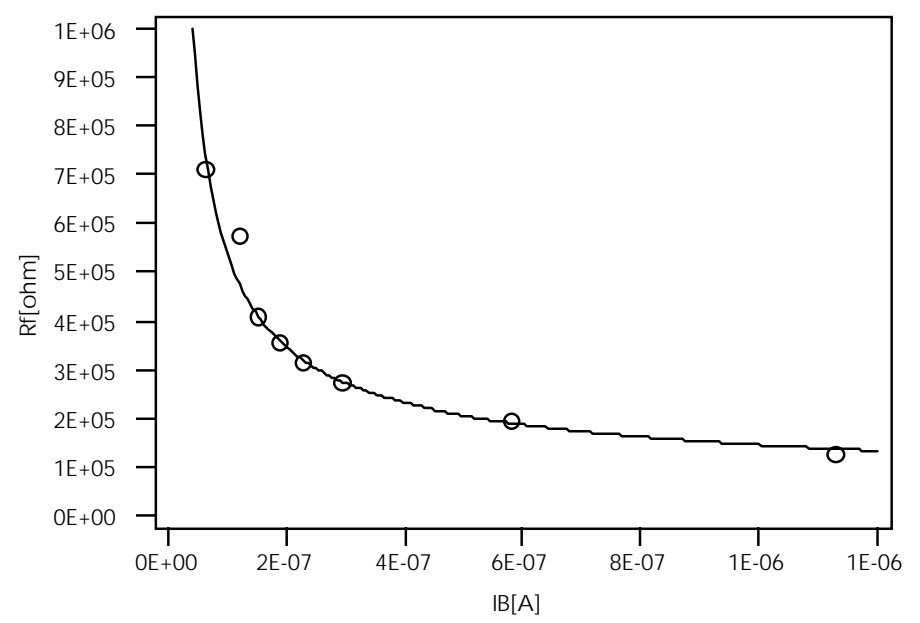

Fig. 7 Measured transresistance as function $I_{f}$. Result of the fit indicates that for $I_{f}$ above $200 \mathrm{nA}, R_{f}$ is not linear with $I_{f}$ but is a power function, because $\mathrm{MP}_{f}$ is working in medium inversion.

The ENC, shown in Fig. 8, has been measured as a function of the input capacitance for three different feedback currents and at bias current of the input branch of $400 \mu \mathrm{A}$, providing an amplifier transconductance of $7 \mathrm{mS}$. For $C_{I N}=0$, a parallel noise, ENCp $=250$ electrons $\mathrm{rms}$, is measured for the higher feedback resistance of $580 \mathrm{k} \Omega\left(I_{f}=120 \mathrm{nA}\right)$. The measured noise slope is 30 electrons $\mathrm{rms} / \mathrm{pF}$, obtained for a rise time of $45 \mathrm{~ns}$. When the same amplifier parameters are used to make the noise calculation for a charge amplifier followed by a CR$\mathrm{RC}^{2}$ filter, we obtain a noise slope of 32 electrons $\mathrm{rms} / \mathrm{pF}$ and a value of $\mathrm{ENCp}=200$ electrons rms at $C_{I N}=0$. The series-noise difference can be explained by the rise-time variation with the input capacitance, which decreases the measured noise slope. The difference between the calculated and measured parallel noise can be explained by the uncertainty as to the absolute value of the total input capacitance, which in our test set-up is $10 \mathrm{pF} \pm 1.5 \mathrm{pF}$. Taking into account these effects, experimental results and calculation agree very well. 


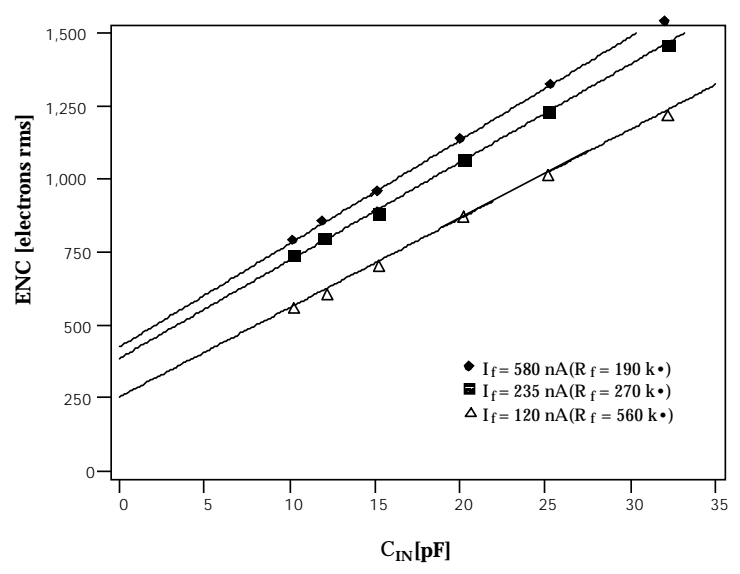

Fig. 8 Experimental noise measurement of the CMOS active feedback amplifer expressed as ENC as function of $C_{I N}$. The measurement is done for three values of $I_{f}$. For $I_{f}=120 \mathrm{nA}(560 \mathrm{k} \Omega)$, the noise slope is $30 \mathrm{el}$. $\mathrm{rms} / \mathrm{pF}$ and $\mathrm{ENC}=250 \mathrm{el} . \mathrm{rms}$ at $C_{I N .}=0 \mathrm{pF}$.

\section{CONCLUSIONS}

A new active feedback technique for transimpedance amplifiers is presented. A CMOS amplifier circuit based on this novel technique has been designed and tested. This circuit employs $\mathrm{n}$-channel and p-channel devices exclusively. The feedback resistance of the amplifier is adjustable from $150-700 \mathrm{k} \Omega$ via a DC current. The phase margin of the amplifier is precisely adjustable via a DC voltage. The measured transimpedance gain is $40 \mathrm{mV}$ for an input charge of 25000 electrons, with a peaking time of $45 \mathrm{~ns}$ for a transresistance of 500 $\mathrm{k} \Omega$. In these conditions, a total noise of 800 electron rms is obtained for an input capacitance of $20 \mathrm{pF}$, making this circuit very promising for low-noise and fast-preamplifier applications. Comparable designs using radiation-hard CMOS and BiCMOS technologies are in development. Preliminary simulations of these versions indicate the same circuit behaviour with a faster rise time.

\section{REFERENCES}

[1] P. Jarron and M. Goyot, A fast current sensitive preamplifier (MSD2) for the silicon microstrip detector, Nucl. Instrum. Methods Phys. Res. A226 (1984) 156-162.

[2] P. D'Angelo, P. Jarron and F. Manfredi, Low noise front-end electronics for microstrip position sensors and vertex detectors for experiments at $\mathrm{TeV}$ energies, Nucl. Instrum. Methods Phys. Res. A241 (1985) 107-114.

[3] W. Dabrowski, G. Bonazzola, P. De Remigis, P. Giubellino, Fast bipolar front-end for binary readout of silicon strip detectors, Nucl. Instrum. Methods Phys. Res. A350 (1994) 548-555.

[4] F. Anghinolfi, P. Aspell, M. Campbell, E.H.M. Heijne, P. Jarron, G. Meddeler and J.C. Santiard, ICON, a current mode preamplifier in CMOS technology for use with high rate particle detectors, IEEE Nucl. Sci. Trans. on Nuclear Science, Vol. 40, No. 3, June 1993.

[5] I. Kipnis, H. Spieler and T. Collins, A bipolar analog front-end integrated circuit for the SDC silicon tracker, IEEE Trans. on Nuclear Science, Vol. 40, No. 2, p. 872, August 1993. 
[6] F.M. Newcomer, S. Tedja. R. Van Berg, J. Van Der Spiegel and H.H Williams, A fast, low power, amplifier-shaper-discriminator for high rate straw tracking systems, IEEE Trans. on Nuclear Science, Vol. 40, No. 4, p. 630, August 1993.

[7] E. Barberis, N. Cartiglia, D.E. Dorfan, J. Rahn, E.N. Spencer, M. Wilder, A fast amplifier-comparator integrated circuit for silicon strip detectors, IEEE Trans. on Nucl. Sci., Vol. 40, No. 4, p. 740, August 1993.

[8] Study of a CMOS-JFET-Bipolar radiation hard analog-digital technology suitable for high energy physics electronics, M. Dentan, IEEE Trans. on Nucl. Sci. Vol. 40, No. 6, p. 1555.

[9] F. Faccio, M. Bianchi, G. Borel, M. Fornasari, E.H.M. Heijne, P. Jarron, G. Rossi and J. Redolfi, Noise characterisation of transistors in a $1.2 \mu \mathrm{m}$ CMOS-SOI technology up to a total dose of $12 \mathrm{Mrad}$, IEEE Trans. on Nucl. Sci., Vol. 41, No. 6, Dec. 1994.

[10] S.L. Thomas, M.J. French, P.Seller, S. Bouvier, G. Hall, M. Millmore, D.M. Raymond, E. Nygard, K. Yoshioka. Measurements of a radiation hardened process, Harris AVLSIRA, Nucl. Instrum. Methods Phys. Res. A342 (1994) 164-168.

[11] Carver Mead, Analog VLSI and neural systems, 1989, Addison-Wesley. 